\title{
Finite-Size Scaling as a Way to Probe Near-Criticality in Natural Swarms
}

\author{
Alessandro Attanasi, ${ }^{1,2}$ Andrea Cavagna, ${ }^{1,2,3, \dagger}$ Lorenzo Del Castello, ${ }^{1,2}$ Irene Giardina, ${ }^{1,2,3}$ Stefania Melillo, ${ }^{1,2, *}$ \\ Leonardo Parisi, ${ }^{1,4}$ Oliver Pohl,${ }^{1,2}$ Bruno Rossaro, ${ }^{5}$ Edward Shen, ${ }^{1,2}$ Edmondo Silvestri, ${ }^{1,6}$ and Massimiliano Viale ${ }^{1,2}$ \\ ${ }^{1}$ Istituto Sistemi Complessi, Consiglio Nazionale delle Ricerche, UOS Sapienza, 00185 Rome, Italy \\ ${ }^{2}$ Dipartimento di Fisica, Università Sapienza, 00185 Rome, Italy \\ ${ }^{3}$ Initiative for the Theoretical Sciences, The Graduate Center, 365 Fifth Avenue, New York, New York 10016 USA \\ ${ }^{4}$ Dipartimento di Informatica, Università Sapienza, 00198 Rome, Italy \\ ${ }^{5}$ DeFENS, Università degli Studi di Milano, 20133 Milano, Italy \\ ${ }^{6}$ Dipartimento di Fisica, Università di Roma 3, 00146 Rome, Italy
}

(Received 17 April 2014; revised manuscript received 9 July 2014; published 1 December 2014)

\begin{abstract}
Collective behavior in biological systems is often accompanied by strong correlations. The question has therefore arisen of whether correlation is amplified by the vicinity to some critical point in the parameters space. Biological systems, though, are typically quite far from the thermodynamic limit, so that the value of the control parameter at which correlation and susceptibility peak depend on size. Hence, a system would need to readjust its control parameter according to its size in order to be maximally correlated. This readjustment, though, has never been observed experimentally. By gathering three-dimensional data on swarms of midges in the field we find that swarms tune their control parameter and size so as to maintain a scaling behavior of the correlation function. As a consequence, correlation length and susceptibility scale with the system's size and swarms exhibit a near-maximal degree of correlation at all sizes.
\end{abstract}

DOI: 10.1103/PhysRevLett.113.238102

PACS numbers: 87.18.Vf, 05.65.+b, 47.54.-r, 87.23.Cc

Intriguing evidence has been presented in the past few years suggesting that some biological systems are close to criticality, namely to a special point in the control parameters space characterized by unusually large correlation and susceptibility [1]. Although reminiscent of self-organized criticality (SOC), this phenomenon is quite distinct, in that it does not appear to be as essentially dynamical as SOC, and it finds its natural description in terms of steady-state ensemble distributions [1]. In all studies where the control parameter has been reported, though, its value has invariably been the result of inference through a model [2]. Inference is potentially prone to the problem of data undersampling and therefore the alleged vicinity of the inferred control parameter to a critical point has been questioned [3]. Even though direct experimental measurements of long-range correlations and scaling laws provide inference-free evidence [4-6], one could still object that conservation laws plus off-equilibrium dynamics can produce long-range correlations generically, namely without the need to tune the control parameter [7]. Therefore, the lack of a direct experimental measurement of the actual vicinity of the control parameter to its critical value is a major missing piece of evidence in the debate about criticality in biological systems. To make things even more complicated, there cannot be just one critical value of the control parameter. The critical point is sharply defined only in the thermodynamic limit. However, all biological groups have finite size, $N$, which is often quite different from group to group. The only finite-size remnant of criticality is the peak of some susceptibility, whose position approaches the bulk critical point for large sizes [8,9]. Thus, at finite size, the effective critical value of the control parameter depends on $N$. A value of the control parameter that makes a small system "critical" will be quite off-critical for a much larger system, and vice versa. For example, a very small Ising model at the bulk critical temperature is in fact deeply magnetized, with very small connected correlation. Hence, the parameters of a biological system cannot simply be tuned to their bulk critical value, as this value would not be critical at all for systems with small $N$. In order to observe critical behavior, the control parameters must depend on the system's size. Therefore, in the discussion about criticality in biological systems we lack two crucial pieces of evidence: (i) a direct experimental measurement of the control parameter (as opposed to model-based inference) and (ii) experimental evidence that in systems of different size $N$ the control parameter varies with $N$ in such a way to keep the system always close to the maximum of the susceptibility. The aim of this Letter is to address these two points.

We study wild swarms of midges in the field (Diptera: Chironomidae and Diptera: Ceratopogonidae) by reconstructing the $3 d$ trajectories of individual insects within swarms ranging from 100 to 600 individuals $[10,11]$. The $3 d$ reconstruction of a swarm is shown in Fig. 1(a) and in the Supplemental Material, video 1 [12]. Swarms of diptera have been also studied in [26-29]. Swarms are in a disordered phase, characterized by a low value of the alignment order parameter (average polarization, $\Phi=0.2$ see Table I in [12]), but at the same time swarms exhibit 
(a)

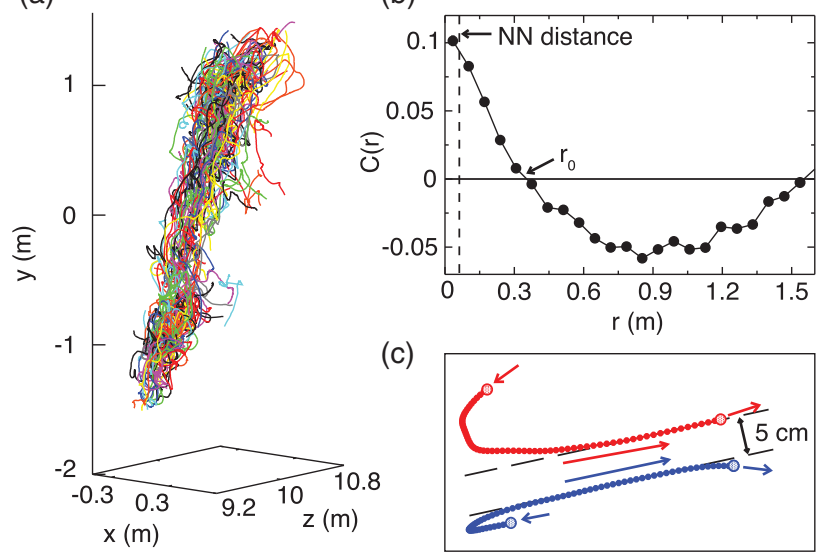

FIG. 1 (color). (a) 3D trajectories for swarm 20120907_A1, $N=169$. (b) Velocity correlation function. The correlation length, $\xi \sim r_{0}$, is much larger than the nearest-neighbor distance. The correlation is averaged over the whole time acquisition. (c) Alignment event between two midges (real trajectories).

significant directional correlations between individuals [10]. For each configuration, we define the equal-time, connected velocity correlation function as follows $[5,10]$ :

$$
C(r)=\frac{\sum_{i \neq j}^{N} \overrightarrow{\delta \varphi}_{i} \cdot \dot{\delta \varphi_{j}} \delta\left(r-r_{i j}\right)}{\sum_{i \neq j}^{N} \delta\left(r-r_{i j}\right)},
$$

where $\delta \vec{\varphi}_{i}$ is the dimensionless velocity fluctuation, $\delta \vec{\varphi}_{i}=\delta \vec{v}_{i} / \sqrt{(1 / N) \sum_{k}\left(\delta \vec{v}_{k}\right)^{2}}$, and $\delta \vec{v}_{i}$, is calculated by subtracting from the individual velocity $\vec{v}_{i}$ the contribution of the instantaneous global translation, rotation, and dilatation of the swarm (see the Supplemental Material [12] for details). The point where the correlation function first reaches zero, $C\left(r_{0}\right)=0$, is a finite-size proxy of the correlation length, $\xi$ [12]. The integrated correlation,

$$
\chi=\frac{1}{N} \sum_{i \neq j}^{N} \overrightarrow{\delta \varphi_{i}} \cdot \overrightarrow{\delta \varphi_{j}} \theta\left(r_{0}-r_{i j}\right),
$$

is a finite-size proxy of the standard susceptibility computed from the fluctuations of the order parameter [8] (see [12]) and for this reason we refer to it as the "susceptibility." In a noninteracting system we find, on average, $\chi=0.1$ [10]. In natural swarms $\chi \in[0.12: 5.6]$ (see Table I in the Supplemental Material [12]). Hence, the most correlated swarms have a susceptibility over 50 times larger than that of a noninteracting system. Large velocity correlations strongly suggest that an effective alignment interaction is present in swarms. Indeed, when two midges get closer than their metric interaction range (which is of the order of a few centimeters $[10,30]$ ) they tend to align their direction of motion [Fig. 1(c)].

Effective alignment, strong correlation, and low-order parameter are phenomena that find a natural interpretation within Vicsek's model of collective motion [31]. In this model each individual aligns its velocity to that of neighbors within a metric interaction range, $\lambda$. At fixed low noise, the model exhibits a transition from a disordered phase (swarming) at low density, to an ordered phase (flocking) at high density. This density-driven transition is controlled by the parameter $x=r_{1} / \lambda$, namely the nearestneighbor distance, $r_{1}$, rescaled by the interaction range, $\lambda$. Hence, there is a value $x_{c}$ of the control parameter below which spontaneous alignment emerges [31-33]. In the case of midges we do not know the interaction range $\lambda$. However, it was suggested in [10] that the interaction between midges is acoustic, so that $\lambda$ is likely to be proportional to the body length, $l$. For this reason we can define the control parameter of swarms as $x=r_{1} / l$ (see the Supplemental Material [12]).

The bulk nature of the Vicsek transition is first order [33]; however, unless $N$ is very large, a pseudo-secondorder phenomenology is observed, where all correlation markers (as $\xi$ and $\chi$ ) peak at the transition [31,34]. This ordering transition has been indeed observed in animal groups [35]. Natural swarms of midges always exhibit low polarization and therefore live on the low-density, disordered side of the transition. Yet correlations are strong, suggesting that natural swarms are not too far from the transition. To investigate more precisely this point, though, we need a finite size scaling approach.

Finite-size scaling (FSS) has been studied in great detail both in equilibrium [8,9] and in off-equilibrium [36] systems. In the case of the Vicsek model, a signature of the first-order nature of the transition occurs above a crossover size that is typically very large (e.g., $N \sim 10^{6}$ in $3 d$; see Ref. [33] and the Supplemental Material [12]). This means that below this size there exists a wide regime (the one relevant for swarms) where FSS holds. This has been shown for the $2 d$ Vicsek model in Refs. [31,34]. Here we present evidence of FSS also in $3 d$ [Fig. 2(a)]: the susceptibility, $\chi$, has a peak at a pseudocritical value $x_{\max }(N)$ of the control parameter, marking the finite-size crossover from a large $x$ disordered phase to a low $x$ ordered one. For larger $N$ the peak becomes sharper and shifts according to the FSS equation, $x_{\max }(N)=x_{c}+1 / N^{1 / 3 \nu}$, where $\nu$ is the critical exponent of the correlation length $\xi$ and $x_{c}$ is the bulk critical point. The scaling variable (at fixed noise) is thus $y=\left(x-x_{c}\right) N^{1 / 3 \nu}$, so that we expect susceptibility and correlation length to behave as $\chi=N^{\gamma / 3 \nu} f(y)$ and $\xi=L g(y)$, where $f$ and $g$ are scaling functions. The scaling behavior of the susceptibility in the $3 d$ Vicsek model is quite satisfying [Fig. 2(a), inset], giving $x_{c}=0.421 \pm 0.002$. Identical results hold in the more realistic case of a Vicsek model with harmonic confinement, which mimics the presence of the marker (see the Supplemental Material [12]).

We can now use Fig. 2(a) as a map to interpret our experimental data. In the disordered phase, $x>x_{c}$, the rotational symmetry is unbroken (low polarization); hence, 
(a)

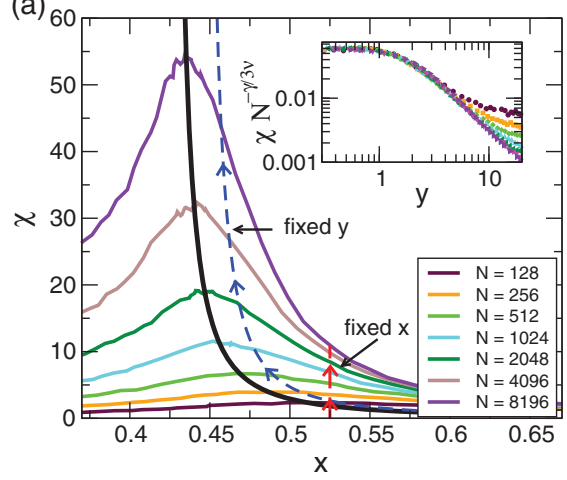

(b)

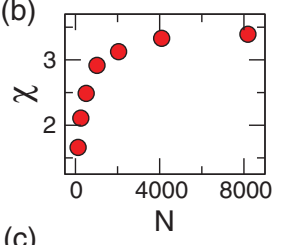

(c)

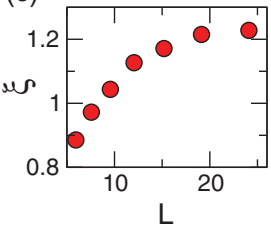

FIG. 2 (color). (a) Vicsek model in $3 d$. Susceptibility $\chi$ as a function of the rescaled nearest-neighbor distance, $x=r_{1} / \lambda$ for different swarm sizes, $N$. The maximum of $\chi$ occurs at the finite-size critical point, $x_{\max }(N)$, marked by the black line. Inset: rescaled susceptibility $\chi N^{-\gamma / 3 \nu}$ vs scaling variable $y=$ $\left(x-x_{c}\right) N^{1 / 3 \nu}$. (b) Susceptibility as a function of $N$ at fixed $x$. (c) Correlation length as a function of the linear system size, $L$, at fixed $x$. By increasing $N$ (and $L$ ) at fixed value of $x$ we are moving along the red path in panel (a), so that we end up being further away from the position of the maximum of $\chi$. Simulations have been performed using the Vicsek update rule in $3 d$ : $\left.\vec{v}_{i}(t+1)=v_{0} \mathcal{R}_{\eta}\left(\sum_{r_{i j}<\lambda} \vec{v}_{j}(t)\right) / \mid \sum_{r_{i j}<\lambda} \vec{v}_{j}(t)\right) \mid ; \quad \vec{r}_{i}(t+1)=\vec{r}_{i}(t)+$ $\vec{v}_{i}(t)$, where $\mathcal{R}_{\eta}$ is a random uniform rotation in $[-2 \pi \eta, 2 \pi \eta]$. $v_{0}=0.05, \lambda=1, \eta=0.45$ (see the Supplemental Material [12]).

no Goldstone mode is present [8] and the Vicsek model has a susceptibility and a correlation length which are finite in the infinite $N$ limit. Hence, by increasing $N$ at fixed $x$ [red path in Fig. 2(a)], $\chi$ initially grows, but then it saturates to its finite bulk value for large $N$ [Fig. 2(b)]. Consider two systems of sizes $L_{1}<L_{2}$, both of which are smaller than the bulk correlation length, $\xi_{\infty}$. When we increase the size of the group, passing from $L_{1}$ to $L_{2}$ all the individuals that we are adding are within a distance $\xi_{\infty}$ from each other and they are therefore strongly correlated; hence, in this regime the finite-size $\xi$ grows with $L$ [Fig. 2(c)] and $\chi$ with $N$ [Fig. 2(b)]. On the contrary, when $L>\xi_{\infty}$ an increase of the size amounts to adding particles statistically uncorrelated from each other, so that both $\xi$ and $\chi$ must saturate with the size [Figs. 2(b) and 2(c)].

In natural swarms, however, we do not observe a saturation of the susceptibility $\chi$, nor of the correlation length $\xi$, with the system's size. Instead, experimental data show that the susceptibility scales with $N$ and the correlation length scales with $L$ up to our largest sizes [Figs. 3(a) and $3(b)]$. There is nothing wrong with the aforementioned explanation, though: the saturation of $\chi$ and $\xi$ for large $N$ should only occur at fixed value of the control parameter, $x$. Swarms, however, do not have a fixed value of $x$, but pick up their own values of $N$ and $x$. The fact that $\chi$ and $\xi$ show no hint of saturation suggests that when $N$ gets larger, $x$ decreases, as if swarms were following the peak of the susceptibility, yet remaining on the disordered side of the transition. This near-critical behavior occurs when the

control parameter $x$ and the system's size $N$ are related in such a way to keep constant the scaling variable, $y=$ $\left(x-x_{c}\right) N^{1 / 3 \nu}$, which is what happens along the blue path in Fig. 2(a). In this case, the following relations must hold:

$$
\begin{gathered}
x \sim x_{c}+N^{-1 / 3 \nu}, \\
\chi \sim N^{\gamma / 3 \nu}, \\
\xi \sim L .
\end{gathered}
$$

Equation (3) defines the near-critical region: it is this mutual readjustment of $x$ and $N$ that keeps the system scale-free, hence giving Eqs. (4) and (5). Although the scatter is significant, the experimental data are compatible with Eqs. (3)-(5) [Figs. 3(a)-3(c)]. In particular, we observe a correlation between control parameter $x$ and size $N$ [Fig. 3(c)]. This is the most prominent evidence that the data are in the near-critical region: not only the correlation in swarms is scale-free $(\xi \sim L, \chi \sim N)$, but a change in the size $N$ of the group is accompanied by a change in the control parameter $x$ so as to compensate finite-size effects and keep the system scale-free correlated. If $(x, N)$ are in the nearcritical region defined by (3), the susceptibility must depend on $x$ as

$$
\chi \sim \frac{1}{\left(x-x_{c}\right)^{\gamma}},
$$

which is the black line in Fig. 2(a). Again, the scatter is large, but we can see from Fig. 3(d) that the susceptibility of swarms indeed grows on decreasing the rescaled nearestneighbor distance $x$, with no evidence of a maximum, so that (6) does a fair job in fitting the data. In the lower panels of Fig. 3 we report the behavior of the $3 d$ Vicsek model in the near-critical region, namely in the region defined by a constant value of the scaling variable $y=\left(x-x_{c}\right) N^{1 / 3 \nu}$ [blue path in Fig. 2(a)]. The similarity with natural swarms is quite satisfying.

Even though we have data for smaller swarms $(N \ll 100)$, we find that surface effects are too strong for these cases and that the statistical approach we use here is not justified anymore. On the other hand, at the moment it is technically hard to record swarms with $N \gg 10^{3}$. The span of our experimental data is therefore limited and different fits would work equally well. Hence, the value of the critical exponents is far from conclusive (also see the Supplemental Material [12]). Therefore, we simply claim that data are compatible with the FSS scenario of the Vicsek model and that the data show scaling. It is important to note that the result that natural swarms live in the near-critical region at the edge of an ordering transition is independent of the data fit.

What distinguishes our results about near-criticality from previous studies is that: (i) we measure, rather than infer, the control parameter, and (ii) we do not simply find a generic vicinity of the control parameter to its bulk critical 

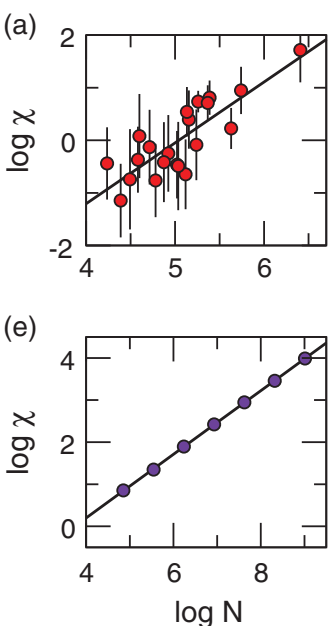

(b)

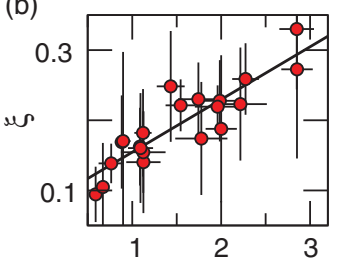

(f)

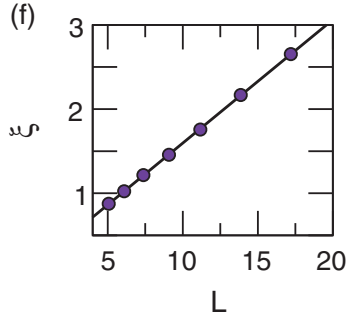

(c)

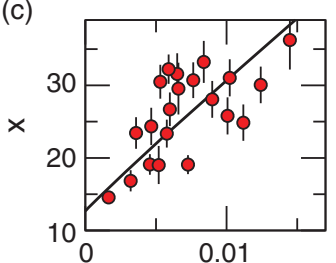

(g)

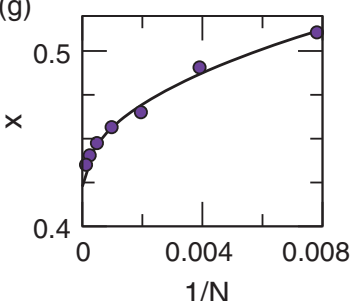

(d)

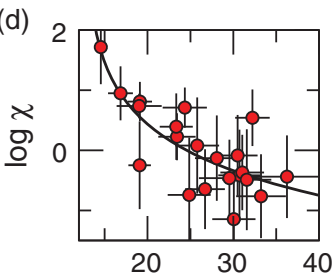

(h)

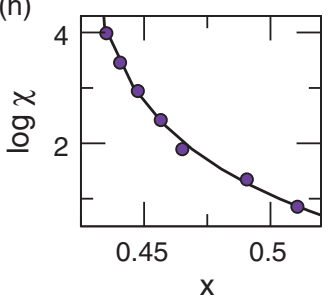

FIG. 3 (color). Top: natural swarms data. Bottom: $3 d$ Vicsek model in the critical region. (a) Susceptibility as a function of the number of midges, $N\left(P\right.$ value $\left.=3.0 \times 10^{-6}\right)$. (b) Correlation length, $\xi$, as a function of the linear system size, $L\left(P\right.$ value $\left.=1.0 \times 10^{-7}\right)$. Both susceptibility and correlation length show no saturation for large systems. (c) Control parameter $x$ as a function of $N$ ( $P$ value $\left.=1.4 \times 10^{-3}\right)$. (d) Susceptibility as a function of the control parameter, $x\left(P\right.$ value $\left.=6.9 \times 10^{-5}\right)$. Each point corresponds to a different swarm averaged over time (error bars are standard deviations). Lower panels (e),(f),(g),(h): same quantities as in the upper panels, but calculated for the Vicsek model in the critical region, defined by a fixed value of the scaling variable $y$. This means that, unlike in Figs. 2(b) and 2(c), in panels 3(e) and 3(f) we are changing both $N$ and $x$ according to Eq. (3) [blue path in Fig. 2(a)]. Lines are fit to Eqs. (3)-(6). For $3 d$ Vicsek we obtain: $\nu=0.75 \pm 0.02, \gamma=1.6 \pm 0.1$, and $x_{c}=0.421 \pm 0.002$, not far from the $3 d$ Heisenberg exponents [37]. For natural swarms we obtain, $\nu=0.35 \pm 0.1, \gamma=0.9 \pm 0.2$, and $x_{c}=12.5 \pm 1.0$. In natural swarms $L$ and $\xi$ are expressed in meters, while both $\chi$ and $x$ are dimensionless.

value, but we actually observe a mutual adjustment of control parameter and system's size that grants the system scale-free correlations. This second result seems to rule out the generic scale invariance of [7]. Note that when $N$ is rather small the pseudocritical value of the control parameter, $x_{\max }(N)$, can be quite far from the bulk critical point, $x_{c}$. What matters is the balance between $N$ and $x$, not just the vicinity to $x_{c}$. When dealing with biological groups, where $N$ is never as large as in condensed matter, it is essential to keep in mind this finite-size scaling description of criticality. It is the pair $(x, N)$ that needs to be in the scaling region, not simply the control parameter.

There are two different ways of interpreting our results. The first possibility is that, given the size $N$, the control parameter $x$ is tuned close to $x_{\max }(N)$, so that the group is endowed with large correlation. This mechanism requires individuals in the group to be able to assess global correlation by means of some local proxy, so that the control parameter $x$ can be readjusted if $N$ is varied. There is, however, an other interpretation. Instead of asking what is the optimal $x$ given $N$, we can ask what is the optimal $N$ given $x$. For each value $x$ of the control parameter, there is an optimal size $N_{\max }(x)$ [obtained by inverting Eq. (3)] for which the maximum of the curve $\chi(x)$ occurs precisely at that $x$ [Fig. 2(a)]. Hence, it is possible that given the control parameter, $x>x_{c}$, a group grows up to its maximum sustainable size, $N_{\max }(x)$. For all values of $N<N_{\max }(x)$ the system is in the ordered phase, where the correlation length scales with the system's size (due to Goldstone's mode). Hence the swarm can grow maintaining a constant level of relative correlation, $\xi / L$. On the contrary, for $N>N_{\max }(x)$, the group would lose correlation with increasing size $(\xi / L \rightarrow 0)$, leading to statistically independent clusters and a deterioration of collective response. Swarms have a mating purpose and male are naturally attracted to them [38]. Hence, an aggregation mechanism that leads to a maximum sustainable size is plausible. This might also explain why swarms do not order: the tendency to maximize the size of the group without decreasing correlation may drive the swarm away from the ordered phase; see also the Supplemental Material [12].

Scale-free correlations similar to those we have reported here for midges have been found in biological groups as diverse as bird flocks [5] and bacteria clusters [39]. Novel experiments trying to link correlation to collective response are needed to understand why correlation seems to be so widespread in biological systems.

We thank William Bialek, Yariv Kafri, Dov Levine, and Victor Martin-Mayor for discussions. This work was supported by IIT grant Seed Artswarm, ERC-StG Grant No. 257126, and US-AFOSR Grant No. FA95501010250 (through the University of Maryland).

* Corresponding author. stefania.melillo79@gmail.com †andrea.cavagna@roma1.infn.it

[1] W. Bialek and T. Mora, J. Stat. Phys. 144, 268 (2011). 
[2] T. Hastie, R. Tibshirani, and J. Friedman, The Elements of Statistical Learning (Springer, Berlin, 2009).

[3] I. Mastromatteo and M. Marsili, J. Stat. Mech. (2011) P10012.

[4] A. Clauset, C. R. Shalizi, and M. E. J. Newman, SIAM Rev. 51, 661 (2009).

[5] A. Cavagna, A. Cimarelli, I. Giardina, G. Parisi, R. Santagati, F. Stefanini, and M. Viale, Proc. Natl. Acad. Sci. U.S.A. 107, 11865 (2010).

[6] T. Mora, A. M. Walczak, W. Bialek, and C. G. Callan, Proc. Natl. Acad. Sci. U.S.A. 107, 5405 (2010).

[7] G. Grinstein, D. H. Lee, and S. Sachdev, Phys. Rev. Lett. 64, 1927 (1990).

[8] D. J. Amit and V. Martin-Mayor, Field Theory, The Renormalization Group and Critical Phenomena, 3rd ed. (World Scientific, Singapore, 2005).

[9] Finite Size Scaling and Numerical Simulations of Statistical Systems, edited by V. Privman (World Scientific, Singapore, 1990).

[10] A. Attanasi et al., PLoS Comput. Biol. 10, e1003697 (2014).

[11] A. Attanasi et al., arXiv:1305.1495.

[12] See Supplemental Material at http://link.aps.org/ supplemental/10.1103/PhysRevLett.113.238102, which includes Refs. [13-25], for a video of the 3d-retrieved trajectories, and for a detailed description of the experiment, of the connected correlation and susceptibility, and of Vicsek model.

[13] P. H. Langton and L. C. V. Pinder, Keys to the Adult Male Chironomidae of Britain and Ireland (Freshwater Biological Association, Ambleside, 2007), Vol. I and Vol. II.

[14] J. J. Kieffer, Faune de France: Diptères Nématocŕes Piqueurs: Chironomidae, Ceratopogoninae (Lechevalier, Paris, 1925), Vol. 11.

[15] P. Dominiak, Polish J. Entomol. 81, 211 (2012).

[16] M. Ballerini et al., Proc. Natl. Acad. Sci. U.S.A. 105, 1232 (2008).

[17] A. Czirok, M. Vicsek, and T. Vicsek, Physica (Amsterdam) 264A, 299 (1999).

[18] M. Nagy, I. Daruka, and T. Vicsek, Physica A (Amsterdam) 373, 445 (2007).

[19] J. A. Downes, Trans. R. Entomol. Soc. London 106, 213 (1955).

[20] A. Okubo, Adv. Biophys. 22, 1 (1986).
[21] S. Butail, N. C. Manoukis, M. Diallo, J. M. Ribeiro, and D. A. Paley, Journal of medical entomology 50, 552 (2013).

[22] J. Toner and Y. Tu, Phys. Rev. E 58, 4828 (1998).

[23] W. Bialek, A. Cavagna, I. Giardina, T. Mora, O. Pohl, E. Silvestri, M. Viale, and A. M. Walczak, Proc. Natl. Acad. Sci. U.S.A. 111, 7212 (2014).

[24] B. G. McKie and P. S. Cranston, Can. J. Zool. 83, 553 (2005).

[25] M. V. Fedorova and R. D. Zhantiev, Entomological Revue 88, 521 (2009).

[26] A. Okubo and H.C. Chiang, Researches on population ecology 16, 1 (1974).

[27] E. A. Shinn and G. E. Long, Environmental entomology 15, 355 (1986).

[28] N. C. Manoukis, A. Diabate, A. Abdoulaye, M. Diallo, A. Dao, A. S. Yaro, J. M. C. Ribeiro, and T. Lehmann, Journal of medical entomology 46, 227 (2009).

[29] D. H. Kelley and N.T. Ouellette, Sci. Rep. 3, 1073 (2013).

[30] M. V. Fyodorova and A. I. Azovsky, Journal of insect behavior 16, 295 (2003); C. Pennetier, B. Warren, K. Roch Dabiré, I. J. Russell, and G. Gibson, Curr. Biol. 20, 131 (2010).

[31] T. Vicsek, A. Czirok, E. Ben-Jacob, I. Cohen, and O. Shochet, Phys. Rev. Lett. 75, 1226 (1995).

[32] B. Gonci, M. Nagy, and T. Vicsek, Eur. Phys. J. Spec. Top. 157, 53 (2008).

[33] H. Chaté, F. Ginelli, G. Gregoire, and F. Raynaud, Phys. Rev. E 77, 046113 (2008).

[34] G. Baglietto and E. V. Albano, Phys. Rev. E 78, 021125 (2008).

[35] J. Buhl, D. J. T. Sumpter, I. D. Couzin, J. J. Hale, E. Despland, E. R. Miller, and S. J. Simpson, Science 312, 1402 (2006); C. Becco et al., Physica (Amsterdam) 367A, 487 (2002); N. C. Makris et al., Science 311, 660 (2006).

[36] S. W. Sides, P. A. Rikvold, and M. A. Novotny, Phys. Rev. Lett. 81, 834 (1998); P. Marcq, H. Chate, and P. Manneville, Phys. Rev. E 55, 2606 (1997).

[37] M. Campostrini, M. Hasenbusch, A. Pelissetto, P. Rossi, and E. Vicari, Phys. Rev. B 65, 144520 (2002).

[38] J. A. Downes, Annu. Rev. Entomol. 14, 271 (1969).

[39] X. Chen, X. Dong, A. Beer, H. L. Swinney, and H. P. Zhang, Phys. Rev. Lett. 108, 148101 (2012). 